\title{
Of microbes and mange: consistent changes in the skin microbiome of three canid species infected with Sarcoptes scabiei mites
}

\author{
Alexandra L. DeCandia* ${ }^{*}$, Kennedy N. Leverett and Bridgett M. vonHoldt
}

\begin{abstract}
Background: Sarcoptic mange is a highly contagious skin disease caused by the ectoparasitic mite Sarcoptes scabiei. Although it afflicts over 100 mammal species worldwide, sarcoptic mange remains a disease obscured by variability at the individual, population and species levels. Amid this variability, it is critical to identify consistent drivers of morbidity, particularly at the skin barrier.
\end{abstract}

Methods: Using culture-independent next generation sequencing, we characterized the skin microbiome of three species of North American canids: coyotes (Canis latrans), red foxes (Vulpes vulpes) and gray foxes (Urocyon cinereoargenteus). We compared alpha and beta diversity between mange-infected and uninfected canids using the KruskalWallis test and multivariate analysis of variance with permutation. We used analysis of composition of microbes and gneiss balances to perform differential abundance testing between infection groups.

Results: We found remarkably consistent signatures of microbial dysbiosis associated with mange infection. Across genera, mange-infected canids exhibited reduced microbial diversity, altered community composition and increased abundance of opportunistic pathogens. The primary bacteria comprising secondary infections were Staphylococcus pseudintermedius, previously associated with canid ear and skin infections, and Corynebacterium spp., previously found among the gut flora of S. scabiei mites and hematophagous arthropods.

Conclusions: This evidence suggests that sarcoptic mange infection consistently alters the canid skin microbiome and facilitates secondary bacterial infection, as seen in humans and other mammals infected with S. scabiei mites. These results provide valuable insights into the pathogenesis of mange at the skin barrier of North American canids and can inspire novel treatment strategies. By adopting a "One Health" framework that considers mites, microbes and the potential for interspecies transmission, we can better elucidate the patterns and processes underlying this ubiquitous and enigmatic disease.

Keywords: Microbiome, Dysbiosis, Sarcoptic mange, Sarcoptes scabiei, Coyotes, Red foxes, Gray foxes, Secondary bacterial infection, Staphylococcus pseudintermedius, Corynebacterium

\section{Background}

Sarcoptic mange has been termed a "ubiquitous neglected disease" [1,2]. Although it afflicts over 100 mammal species on every continent except for Antarctica, numerous questions remain about its pathology [3-6]. A major impediment regards the wide-scale

*Correspondence: decandia@princeton.edu Department of Ecology \& Evolutionary Biology, Princeton University, Princeton, NJ, USA variability that sarcoptic mange exhibits at every level of infection from individuals to populations to species, despite its universal source being Sarcoptes scabiei mites [7].

Canids typify this variation. Considered prominent hosts of mange, many canid species are particularly susceptible due to their den usage and sociality $[3,8,9]$. Yet individuals are not affected uniformly. Host symptoms range from mild pruritus to emaciation, dehydration, crust formation or even death $[4,10-12]$. This 
variation scales to the population and species levels, where sarcoptic mange can exist as an enzootic parasite that imposes persistent, low levels of morbidity, or an epizootic parasite that causes dramatic mortality events in virulent outbreaks $[8,13-20]$.

Amid this variability, it is important to elucidate consistent drivers of morbidity, particularly at the skin barrier. Considered the first line of defense against infection, the skin presents a physical and microbial barrier to invading parasites [21-23]. Upon contact with this barrier, adult females burrow into the skin to feed on host lymph and deposit the next generation of eggs [1, 2]. Often completing their entire life-cycle on the same host, mites and their secretions continuously irritate the skin and elicit severe allergic reactions $[3,4,6]$. Secondary bacterial infection with pathogenic microbes (such as Staphylococcus spp. and Streptococcus spp.) typically follows mite infestation [6, 24]. Mites may even facilitate colonization of opportunistic invaders by transporting harmful bacteria to the host's skin [25] and secreting immune inhibitors into burrows and lesions [26, 27].

To examine the effect of sarcoptic mange on the skin microbiome, Swe et al. [28] experimentally infected pigs (Sus scrofa domesticus) with S. scabiei var. suis and sequenced microbial communities over the course of infection. Mange-infected individuals displayed lower levels of microbial diversity, altered community abundance and increased incidence of Staphylococcus spp. compared to their uninfected counterparts. Similar patterns have been observed in humans, domestic animals and wildlife infected with sarcoptic mange $[1,6,12,29]$, as well as domestic dogs (Canis familiaris) and humans with allergic skin conditions, such as atopic dermatitis [30-34]. This evidence suggests that disrupted microbial communities may play a key role in the pathogenesis of sarcoptic mange.

Given the pervasive variability of this neglected disease, additional studies are needed to assess the universality of these trends. We contributed to these efforts by characterizing the skin microbiome of mange infection across three species of North American canids: coyotes (Canis latrans), red foxes (Vulpes vulpes) and gray foxes (Urocyon cinereoargenteus). Canids present an ideal system for these analyses, as they are among the primary species affected by sarcoptic mange in North America [20]. Due to the divergent evolutionary histories of these three genera, we anticipated speciesspecific differences in microbial community composition of healthy and infected individuals. However, given their similar ecologies, we predicted consistent responses to mange infection that included decreased species richness and altered community abundance favoring pathogenic bacteria.

\section{Methods}

\section{Sample and data collection}

We opportunistically collected samples from coyotes, red foxes and gray foxes admitted to licensed wildlife rehabilitation centers between January 2017 and April 2019. Partnering centers included the Wildlife Rehabilitation Center of Minnesota (Minnesota), Fund for Animals Wildlife Center (California), Janet L. Swanson Wildlife Health Center at Cornell University (New York), Woodlands Wildlife Refuge (New Jersey), PAWS Wildlife Center (Washington) and Tufts Wildlife Clinic (Massachusetts). Critically, samples were collected upon admission to each facility and before treatment with antimicrobials, antivirals, anthelmintics or acaricides. This minimized potential confounding effects of artificial environment (such as indoor facilities or human contact), sampling location or treatment regime.

Sample metadata included sampling date and location, primary reason for admission, species, sex, age, weight and mange status. We assessed mange severity by assigning each individual to a mange class corresponding to the percentage body area that exhibited symptoms, such as lesions, crusts or alopecia. Uninfected individuals were assigned to Mange Class 0, with Mange Class 1 defined as $0-5 \%$ of the body covered, Mange Class 2 by $6-50 \%$ and Mange Class 3 by more than 50\%, following [35].

We collected swabs from five body sites (Fig. 1) that included the external ear, dorsal flank, axilla, groin, and outer back leg. We used a sterile $\mathrm{BBL}^{\mathrm{TM}}$ swab to sample the skin at each body site, rotating the swab tip by $90^{\circ}$ every 10 strokes for a total of 40 swab strokes [30]. Samples were stored at $-80^{\circ} \mathrm{C}$ until DNA extraction.

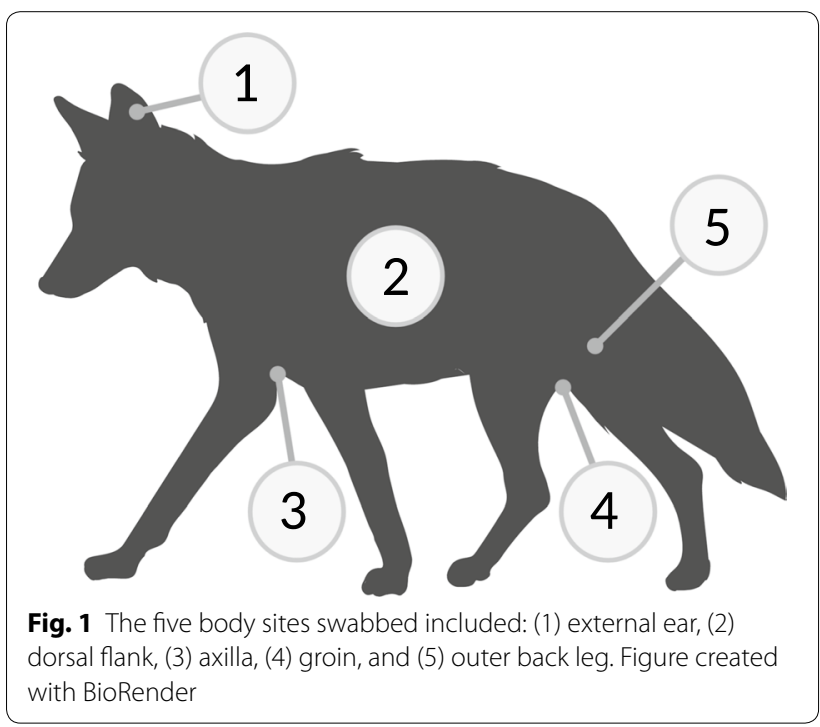




\section{DNA extraction and 165 rRNA V4 sequencing}

We extracted microbial DNA from each swab tip using a modified DNeasy PowerSoil Kit (Qiagen, Hilden, Germany) protocol described in DeCandia et al. [36]. Briefly, we placed each swab tip into a PowerBead tube and used a TissueLyser II (Qiagen, Hilden, Germany) to disrupt samples for two cycles, both $12 \mathrm{~min}$ at 20 shakes/s, with the addition of $60 \mu \mathrm{l}$ of $\mathrm{C} 1$ solution in-between cycles. For the final elution step, we incubated samples at room temperature for $10-15 \mathrm{~min}$ using $60 \mu \mathrm{l}$ of $\mathrm{C} 6$ solution pre-heated to $70{ }^{\circ} \mathrm{C}$. We used sterile swab tips as negative controls during every round of extractions to minimize contamination risk. We subsequently concentrated extracts to $20 \mu \mathrm{l}$ in a Vacufuge and assessed DNA concentrations using a high-sensitivity Qubit ${ }^{\mathrm{TM}}$ fluorometer. We used molecular grade water to standardize samples to $2.5 \mathrm{ng} / \mu \mathrm{l}$ and included low yield samples in subsequent steps.

We amplified and tagged the $16 S$ ribosomal RNA (rRNA) hypervariable 4 (V4) region in each sample through polymerase chain reaction (PCR) using 96 unique combinations of barcoded forward $(n=8)$ and reverse $(n=12)$ primers [37]. As in DeCandia et al. [36], the reaction recipe included $5 \mu \mathrm{l}$ of HiFi HotStart ReadyMix (KAPA Biosystems, Wilmington, USA), $3.2 \mu \mathrm{l}$ of primer mix $(1.25 \mu \mathrm{M})$ and $1.8 \mu \mathrm{l}$ of template DNA. Cycling conditions were as follows: initial denaturation at $94{ }^{\circ} \mathrm{C}$ for $3 \mathrm{~min}$; touchdown cycling for 30 cycles of $94{ }^{\circ} \mathrm{C}$ for $45 \mathrm{~s}, 80-50{ }^{\circ} \mathrm{C}$ for $60 \mathrm{~s}, 72{ }^{\circ} \mathrm{C}$ for $90 \mathrm{~s}$, decreasing $1{ }^{\circ} \mathrm{C}$ each cycle; 12 cycles of $94{ }^{\circ} \mathrm{C}$ for $45 \mathrm{~s}, 50{ }^{\circ} \mathrm{C}$ for $60 \mathrm{~s}$, $72{ }^{\circ} \mathrm{C}$ for $90 \mathrm{~s}$; and final extension of $72{ }^{\circ} \mathrm{C}$ for $10 \mathrm{~min}$. We used Quant-iT ${ }^{\mathrm{TM}}$ PicoGreen $^{\mathrm{TM}}$ dsDNA assays (Invitrogen, Carlsbad, USA) to quantify the PCR products, pooled equal nanograms of each library and selected for amplicons between 300 and $400 \mathrm{nt}$ in length using Agencourt AMPure XP magnetic beads (Beckman Coulter, Brea, USA). We sent final libraries to the Princeton University Genomics Core Facility for paired-end amplicon sequencing $(2 \times 150 \mathrm{nt})$ on an Illumina MiSeq machine (Illumina, San Diego, USA).

\section{Data processing}

We used a paired-end, dual-indexed barcode splitter implemented in Galaxy to demultiplex raw sequencing data, allowing for one nucleotide mismatch between expected and observed barcode sequences [38]. We then imported reads into QIIME 2 v.2019.4 [39, 40] for data filtering. Through the dada2 denoise-paired plugin, we corrected probable sequencing errors, removed chimeras, trimmed low quality bases and merged paired-end reads to identify taxonomic features [41]. We additionally identified operational taxonomic units (OTUs) using de novo-, closed reference- and open reference clustering with qiime vsearch to compare our denoised dataset to more traditional cluster-based methods [42].

\section{Alpha and beta diversity}

We calculated alpha and beta diversity metrics using the core-metrics-phylogenetic and alpha-rarefaction functions in QIIME 2. To correct for differences in read depth, we rarefied samples to 5153 sequences for the full dataset ( $n=125$ samples) and 17,693 sequences for a composite dataset where samples were grouped by individual ( $n=25$ grouped samples). Read depths were chosen to retain all samples for analysis.

To examine within-sample diversity, we calculated the Chao 1 index for species richness and Pielou's evenness metric for species equitability. For between-sample differences, we used fasttree to construct a rooted phylogenetic tree of taxonomic features and calculated unweighted UniFrac distances for species presence, weighted UniFrac distances for species presence and abundance, and the Bray-Curtis dissimilarity index for species abundance. We visualized sample dissimilarity through principal coordinates analysis ( $\mathrm{PCoA}$ ) using the EMPeror plugin [43] and performed significance testing using the Kruskal-Wallis test for alpha diversity metrics and multivariate analysis of variance with permutation (PERMANOVA) for beta diversity differences [44]. Variables of interest included sampling state, species, age, sex, year and mange infection status.

\section{Taxonomic composition and differential abundance testing} We determined the taxonomic composition of each sample using a Naive Bayes classifier trained on Greengenes 13_8 reference sequences trimmed to our $16 S$ rRNA V4 amplicon and clustered at $99 \%$ similarity $[45,46]$. We then used the classify-sklearn function to assign taxonomy to each representative sequence in the dataset [46].

To assess the statistical significance of compositional differences, we used two complementary approaches for differential abundance testing: analysis of composition of microbes (ANCOM) and gneiss balances. ANCOM calculates the log-ratio between pairwise combinations of taxa and sums how many times the null hypothesis is violated [47]. Gneiss calculates log transformed ratios (termed balances) between groups of taxa arranged in a hierarchical tree through correlation clustering [48]. Ordinary least squares (OLS) regression can subsequently be used to test for differences between infection groups. Both analyses require a composition artifact as input, with additional filtering necessary to remove taxonomic features that occur in fewer than 10 samples or have frequencies below 50. We implemented each analysis with our composite dataset where samples were 
grouped by individual, and queried results using the NCBI BLASTn online tool [49].

\section{Results}

\section{Amplicon sequencing and data processing}

We sequenced 153 samples collected from 15 coyotes (mange-infected $=9$, uninfected $=5$, unknown $=1$ ), 13 red foxes (mange-infected $=8$, uninfected $=5$ ) and 2 gray foxes (mange-infected $=1$, uninfected $=1$ ). The full dataset contained 4,397,629 raw reads, which reduced to 3,911,712 sequences after denoising (Additional file 1: Table S1). The denoised dataset contained 11,800 unique taxonomic features, whereas the OTU datasets contained 6137 (de novo), 5456 (closed reference) and 8106 (open reference) features at $97 \%$ percentage identity. Proceeding with the denoised dataset, we removed 28 samples due to incorrect body sites $(n=7)$, treatment prior to sampling $(n=11)$, low read counts $(n=5)$ and unknown mange status $(n=5)$. Our final dataset consisted of 125 samples collected from 12 coyotes (mange-infected $=8$, uninfected $=4$ ), 11 red foxes (mange-infected $=6$, uninfected $=5$ ) and 2 gray foxes (mange-infected $=1$, uninfected $=1$ ).

\section{Uninfected samples cluster by individual rather than body} site

Given repeated measures across individuals ( $n=5$ samples per individual) and body sites $(n=25$ samples per body site) in the denoised dataset, we implemented principal coordinates analysis (PCoA) on uninfected samples to assess whether these factors significantly influenced beta diversity. Across all three distance measures, samples clustered by individual (PERMANOVA; Bray-Curtis, pseudo- $F_{(9)}=2.984, P=0.001$; unweighted UniFrac, $p$ seudo- $F_{(9)}=2.938, P=0.001$; weighted UniFrac, pseudo- $\left.F_{(9)}=3.470, P=0.001\right)$ rather than body site (Bray-Curtis, pseudo- $F_{(4)}=0.781, P=0.997$; unweighted UniFrac, pseudo- $F_{(4)}=0.769, P=0.997$; weighted UniFrac, pseudo- $F_{(4)}=0.950, P=0.574$; Fig. 2, Additional file 2: Figure $\mathrm{S} 1$ ). We therefore grouped samples by individual in downstream analyses to control for statistical relicts of pseudoreplication. Rather than five samples per canid (i.e. one for each body site), each individual was represented by one composite sample that contained all features in their skin microbiome.

We performed significance testing for alpha and beta diversity on our composite dataset to determine which metadata categories were predictive of microbial community structure. Mange infection was consistently the variable most strongly associated with differences in alpha and beta diversity (Additional file 3: Table S2). Although sex appeared significant, further analyses showed nonindependence between sex and mange status (Chi-square

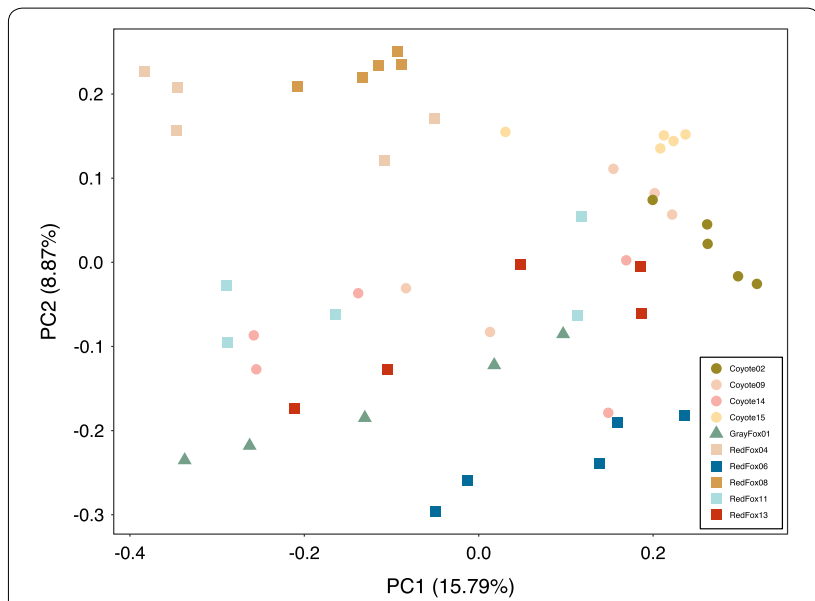

Fig. 2 Principal coordinates analysis (PCOA) of uninfected individuals showed significant clustering by individual (PERMANOVA; pseudo- $F_{(9)}=2.938, P=0.001$ ) rather than body site (pseudo- $F_{(4)}=0.769, P=0.997$ ) using phylogeny-based unweighted UniFrac distances

test, $\left.\mathrm{X}^{2}=4.039, d f=1, P=0.044\right)$, due to a disproportionate number of infected males in the dataset. Notably, test statistics calculated for sex were lower than those calculated for mange infection status (Additional file 3: Table S2). We further performed significance testing on uninfected canids of known sex to see whether male and female canids exhibited different microbial communities. In these analyses, we observed no significant differences in alpha or beta diversity between the sexes (Additional file 4: Table S3). These results were visually confirmed through PCoA (Additional file 5: Figure S2). Considered together, this evidence suggested that mange infection status, rather than sex, was the primary driver underlying differences in microbial community structure. We therefore analyzed the full composite dataset for subsequent analyses and used mange infection status as our variable of interest.

\section{Mange-infected canids exhibit decreased diversity and community evenness across species}

We observed significantly reduced species richness (Kruskal-Wallis test; Chao 1 index, $H=10.711$, $P=0.001$; Fig. 3a) and evenness (Pielou's evenness metric, $H=8.643, P=0.003$; Fig. $3 \mathrm{~b}$ ) in mange-infected individuals. Beta diversity similarly differed by infection group. Measures of species abundance (PERMANOVA; Bray-Curtis, pseudo- $F_{(1)}=3.885, P=0.001$; Fig. 3c), presence (unweighted UniFrac, pseudo- $F_{(1)}=2.211$, $P=0.006$; Additional file 6: Figure S3a), and both presence and abundance considered together (weighted UniFrac, pseudo- $F_{(1)}=4.398, P=0.001$; Additional file 6: Figure S3b) showed significant differences between 


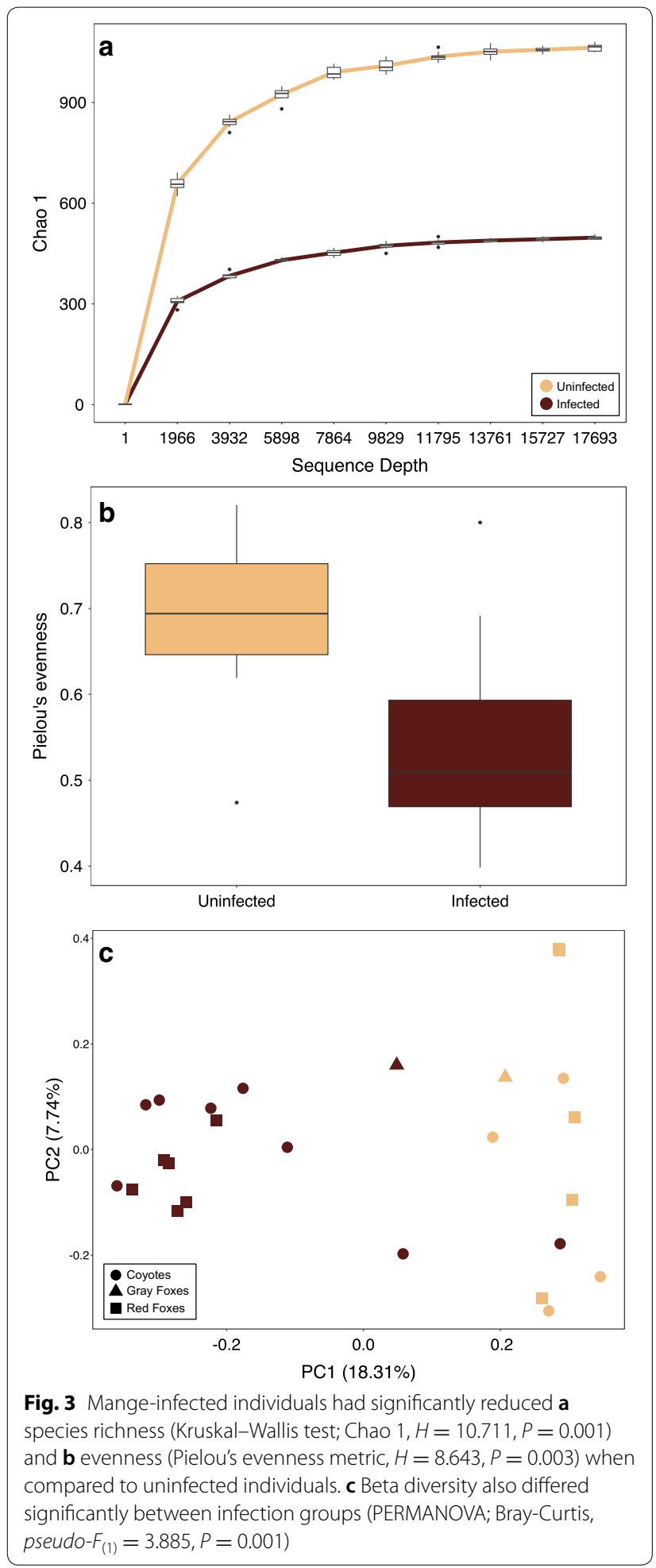

mange-infected and uninfected canids. For all three measures, samples clustered by infection status along PC1, which explained $16.49-29.01 \%$ of the variation.
Taxonomic composition of skin microbial communities confirmed these patterns (Fig. 4). Although variation between individuals was evident, mange-infected canids exhibited higher relative abundance of Actinobacteria (mean \pm standard error, SE, infected $=25.883 \pm 5.183 \%$, uninfected $=12.360 \pm 2.541 \%)$ and Bacilli (mean \pm SE, infected $=35.823 \pm 4.898 \%$, uninfected $=9.154 \pm 2.900 \%$ ), and reduced abundance of "other" taxa (mean $\pm \mathrm{SE}$, infected $=8.262 \pm 1.883 \%$, uninfected $=25.989 \pm 5.346 \%$ ). These results remained consistent even when the dataset was subdivided by species (Additional file 7: Table S4).

\section{Increased abundance of Staphylococcus pseudintermedius and Corynebacterium spp. with mange infection}

Analysis of the composition of microbes (ANCOM) returned one taxonomic feature as consistently and significantly more abundant in mange-infected individuals: feature 3f0449c545626dd14b585e9c7b2d16f4 ( $W=111$; Additional file 8: Figure S4). NCBI BLASTn [49] search results returned high sequence similarity to Staphylococcus pseudintermedius (class Bacilli; Additional file 9: Table S5a). Although not statistically significant, feature e3e89166daa575e51d7a14bc65f11153 exhibited the second highest number of rejected null hypotheses $(W=21)$ and matched Corynebacterium spp. (class Actinobacteria; Additional file 9: Table S5b).

Given the strong effect of mange infection on alpha and beta diversity, we constructed a simple OLS regression model using mange infection status and gneiss balances as the variables of interest. This model explained $9.40 \%$ of the variation observed, and returned two statistically significant balances that contained features with increased taxonomic abundance in mange-infected individuals: y02 and y05 (both $P=0.013$; Fig. 5). After visualizing the tree hierarchy through the Interactive Tree of Life (iTOL) v.3 online tool [50], we found that balance y05 was nested within y02. As a result, both balances pointed towards the same signal: increased proportion of features 3f0449c545626dd14b585e9c7b2d16f4 (mean \pm SE, infected $=0.421 \pm 0.052$, uninfected $=0.032 \pm 0.018)$ and e3e89166daa575e51d7a14bc65f11153 (mean \pm SE, infected $=0.170 \pm 0.040$, uninfected $=0.003 \pm 0.001)$ in mange-infected individuals (Fig. 5a). These features were previously identified as $S$. pseudintermedius and Corynebacterium spp. using NCBI BLASTn, and were clustered with two additional features in the dendrogram relating all taxa: features c2d41dc0a7b8eaedcf4697512aee4427 (identified as Staphylococcus spp.) and 22a5bce17370d6c495f5e83232650ec7 (identified as Streptococcus agalactiae; Fig. 5b). These additional features exhibited higher proportions in infected canids compared to uninfected individuals (Staphylococcus spp. mean $\pm \mathrm{SE}$, 


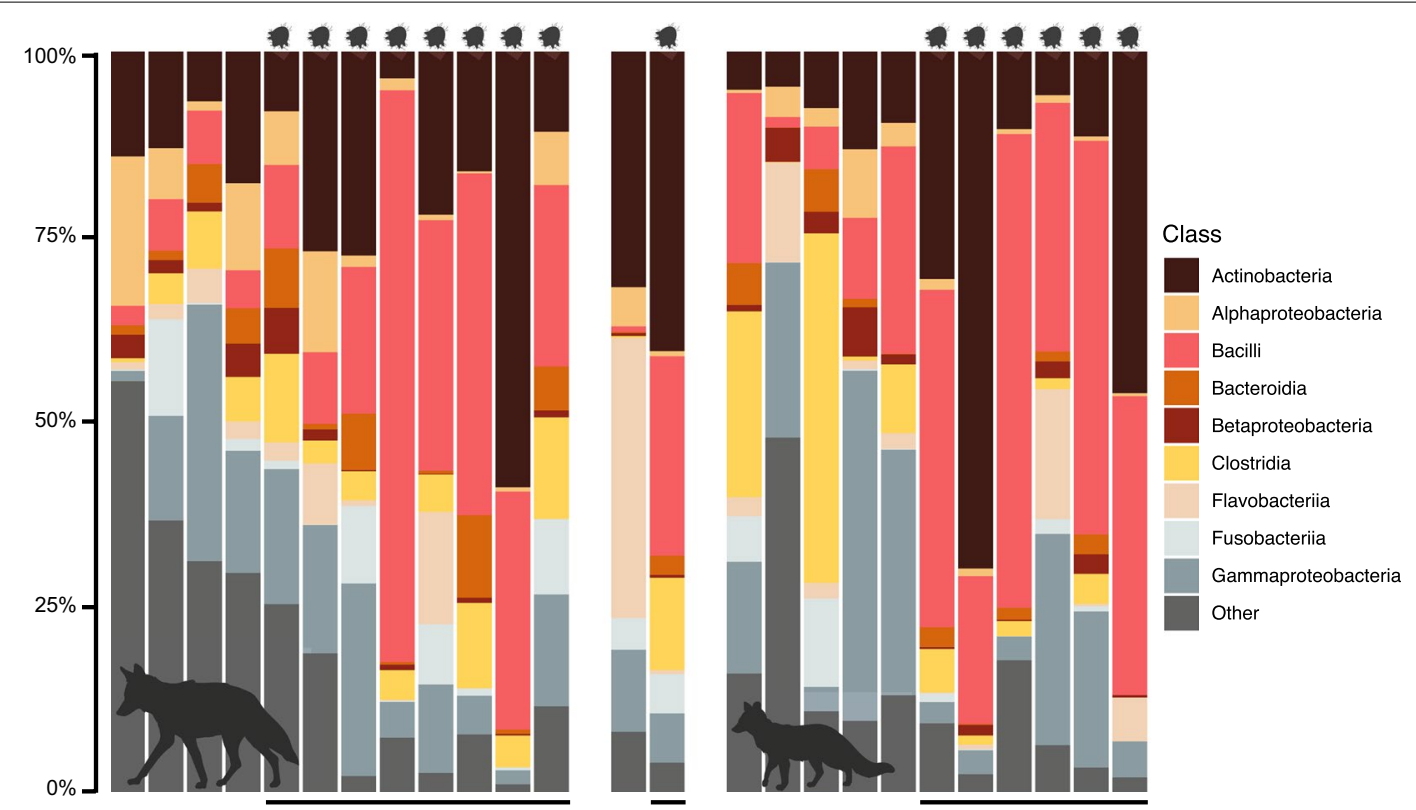

Fig. 4 Taxonomic composition of skin microbial communities for 12 coyotes, 2 gray foxes and 11 red foxes. Black bars (bottom) and mites (top) signify individuals infected with sarcoptic mange. Figure created with BioRender

infected $=0.017 \pm 0.006$, uninfected $=0.001 \pm 0.000 ; S$. agalactiae mean $\pm \mathrm{SE}$, infected $=0.007 \pm 0.003$, uninfected $<0.001 \pm 0.000$ ). Although balance y78 was also statistically significant $(P=0.024)$, its proportions only marginally differed between infection groups, with increased abundance of its component taxa found in uninfected canids.

\section{Discussion}

Sarcoptic mange is among the most widespread diseases affecting mammals on a global scale. Despite recognition since antiquity [2], mange is considered a neglected disease, as there remain numerous questions about its pathology in free ranging wildlife [5]. The interplay between mites and microbes at the skin barrier is one such question, given increasing recognition of the importance of host-associated microbiomes in wildlife health and disease [51-53].

We characterized the skin microbiome of mangeinfected and uninfected canids in three North American species: coyotes, red foxes and gray foxes. Across species, we observed remarkably consistent signatures of mange infection that included reduced diversity, shifted community composition and increased proportion of S. pseudintermedius and Corynebacterium spp. Although samples derived from different species sampled in different states, infection status was the primary driver of microbial community structure in terms of species richness, evenness, presence and relative abundance.
Commensal microbial communities are shaped by a complex milieu of genetic and environmental factors [54, 55]. Although inter-individual variation is pervasive, the host-associated microbiome is thought to exhibit phylosymbiosis between microbes and their hosts over evolutionary timescales $[56,57]$. In a study of small mammals spanning six genera, for example, species identity exerted a far stronger effect on microbial community structure than did local habitat [58]. We therefore anticipated divergence between the skin microbiome of the three focal species, as coyotes, red foxes and gray foxes are in different genera within Canidae. Counter to this expectation, we found minimal differences between skin microbial communities across species, sampling locations, years, sexes and ages. Instead, mange infection status was the primary factor associated with microbial community structure within our dataset. This suggested two primary hypotheses. The first posits that shared evolutionary history and contemporary ecology of these species leads to similar skin microbiomes, as seen in gut microbial communities across families within class Mammalia [59]. The second contends that mange infection alters community composition consistently and dramatically across species, thereby blurring inter-genus distinctions within our relatively small sample set.

Results from this study primarily supported the second hypothesis, although it is likely that evolutionary history, contemporary ecology and mange infection all influenced the observed patterns of microbial diversity. Within the 


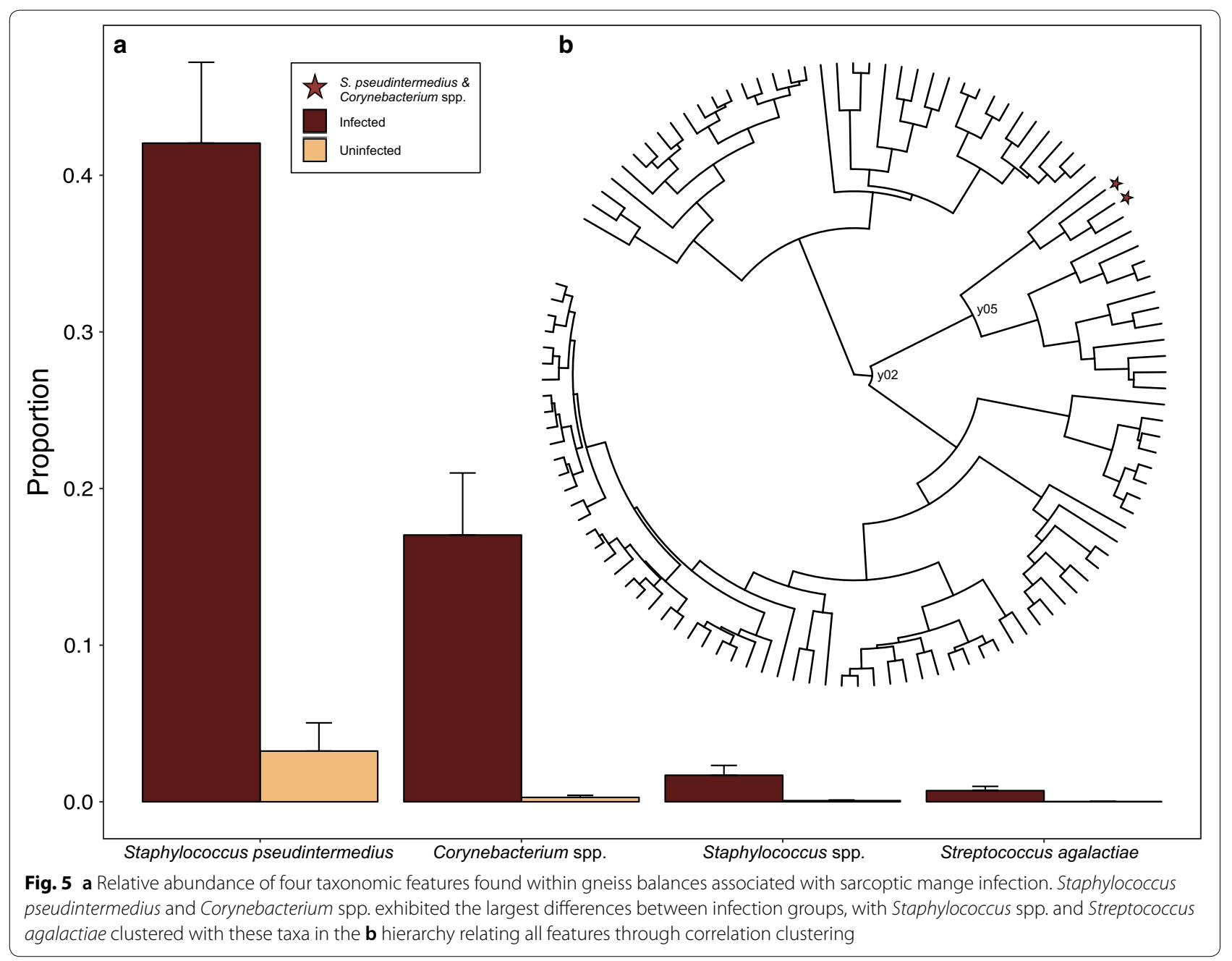

broader context of microbes and mange, reduced microbial variation and increased abundance of opportunistic pathogens is consistent with humans infected with $S$. scabiei var. hominis $[60,61]$, pigs experimentally infected with S. scabiei var. suis [28], Santa Catalina island foxes (Urocyon littoralis catalinae) infected with Otodectes cynotis ear mites [36], and domestic dogs and humans exhibiting allergic skin disorders [31-34]. Although the identity of opportunistic pathogens varied by host species, Staphylococcus spp. and Streptococcus spp. were commonly reported. Mite presence may even facilitate these secondary bacterial infections by secreting proteins that inhibit the mammalian complement system, which is known to be a key player in the immune response against mite and bacterial infections [26, 27, 62, 63]. Mite burrows and host lesions may therefore provide ideal environments for opportunistic pathogens to proliferate.

The primary microbial taxa associated with mange infection in this study included S. pseudintermedius and Corynebacterium spp., with $S$. agalactiae and other
Staphylococcus spp. marginally differing in abundance. Both humans and pigs infected with $S$. scabiei exhibited increased proportion of $S$. aureus [28, 60], with S. pseudintermedius reported in island foxes infected with ear mites [36]. These analogous results present compelling evidence that mite infection is associated with Staphylococcus spp. proliferation across host taxa. Further, increased abundance of $S$. pseudintermedius across four canid species infected with S. scabiei (coyotes, red foxes and gray foxes) and O. cynotis (island foxes [36]) mites suggests that it is an important bacterial taxon within Canidae.

A common canid commensal [64], S. pseudintermedius becomes an opportunistic pathogen when the skin microbiome is disrupted by allergic skin disease, infection or surgery [65-67]. Resultant biofilms can lead to chronic inflammation in domestic dogs, cats (Felis catus) and, to a lesser extent, humans [68], with antibiotic resistant strains emerging across veterinary and medical hospitals $[69,70]$. 
Although less commonly reported across host species, Corynebacterium spp. was detected in skin crusts and S. scabiei mites isolated from pigs with severe mange [28]. Similar bacteria were also isolated from the gastrointestinal tracts of hematophagous arthropods, such as triatomes (Triatoma infestans [71]) and three species of ticks (Ixodes ricinus, Dermacentor reticulatus and Haemaphysalis concinna [72]). This evidence suggests that Corynebacterium spp. may derive from mite bodies, secretions or frass deposited at the site of infection, in addition to canid commensal communities. As with S. pseudintermedius, these bacteria likely benefit from mite inhibition of mammalian complement.

\section{Conclusions}

In the present study, we reported microbial dysbiosis associated with sarcoptic mange infection in three species of North American canids: coyotes, red foxes and gray foxes. Across species, mange was consistently characterized by decreased microbial diversity, altered community composition and increased proportion of opportunistic bacterial infections comprised of S. pseudintermedius and Corynebacterium spp. These additional insights into the pathogenesis of sarcoptic mange may enable novel management of wildlife affected in situ and ex situ [73]. Regarding treatment, acaricides may possess harmful side effects for individuals and the environment, with drug resistance observed in some $S$. scabiei lineages and their concomitant bacterial infections $[1,6,70,74]$. It may become critical to pursue novel avenues of treatment, such as a combination of acaricides and anti- or probiotic therapies, to improve therapeutic outcomes for infected individuals. Insights into mite microbiomes may further provide means of mite control if these communities can be manipulated [71]. Given the ubiquity of this disease and its capacity to infect humans, domestic animals and wildlife, sarcoptic mange presents an ideal candidate for adopting a "One Health" perspective when mitigating its negative effects [5]. Mammalian hosts can be intricately coupled, enabling interspecies transmission when diseased animals approach human settlements in search of resources or shelter, as seen in mange-infected coyotes [75, 76] and red foxes [77]. Although public health concerns are minor due to the lesser severity of zoonotic mange, interspecies transmission between widespread and at-risk species can pose a conservation risk. Thus, identifying consistent drivers of morbidity, such as altered microbial communities, can enable better prediction and mitigation of mange dynamics across host systems.

\section{Supplementary information}

Supplementary information accompanies this paper at https://doi. org/10.1186/s13071-019-3724-0.

Additional file 1: Table S1. Sample metadata and sequence processing statistics for each stage of the dada2 denoising pipeline implemented in QIIME2 v.2019.4.

Additional file 2: Figure S1. Principal coordinates analyses of uninfected individuals showed significant clustering by individual rather than body site using both $\mathbf{a}$ Bray-Curtis and $\mathbf{b}$ phylogeny-based weighted UniFrac distances.

Additional file 3: Table S2. Results from alpha (Kruskal-Wallis test) and beta (PERMANOVA) diversity significance tests performed on the composite dataset.

Additional file 4: Table S3. Results from alpha (Kruskal-Wallis test) and beta (PERMANOVA) diversity significance tests performed on uninfected canids with known sex.

Additional file 5: Figure S2. Principal coordinates analysis of a uninfected individuals (five samples per canid) using phylogeny-based unweighted UniFrac distances and $\mathbf{b}$ all individuals (one composite sample per canid) using Bray-Curtis dissimilarity index show minimal evidence of clustering by sex (females, no outline; males, black outline; unknown, gray outline).

Additional file 6: Figure S3. Principal coordinates analysis showed significant differences between infection groups using both a unweighted and b weighted UniFrac distances.

Additional file 7: Table S4. Relative abundance (mean and standard error) of taxonomic classes in canids grouped by species and infection status. Taxonomic classes include: Bacilli, Actinobacteria, Gammaproteobacteria, Clostridia, Flavobacteriia, Alphaproteobacteria, Fusobacteriia, Bacteroidia, Betaproteobacteria, and other taxa.

Additional file 8: Figure S4. Analysis of the composition of microbes returned one taxonomic feature as consistently and significantly associated with mite infection status: Staphylococcus pseudintermedius (indicated with a red star). Three additional taxa that commonly co-occurred with S. pseudintermedius included Corynebacterium spp. (pink star), Streptococcus agalactiae (red circle) and Staphylococcus spp. (pink circle).

Additional file 9: Table S5. NCBI BLASTn results for the two features exhibiting increased relative abundance in mange-infected individuals: a 3f0449c545626dd14b585e9c7b2d16f4 (class Bacilli) and b e3e89166daa575e51d7a14bc65f11153 (class Actinobacteria).

\section{Abbreviations}

ANCOM: analysis of composition of microbes; BLASTn: basic local alignment search tool for nucleotides; iTOL: Interactive Tree of Life; NCBI: National Center for Biotechnology Information; OLS: ordinary least squares; OTU: operational taxonomic unit; PC1: principal coordinate one; PCOA: principal coordinates analysis; PCR: polymerase chain reaction; PERMANOVA: multivariate analysis of variance with permutation; rRNA: ribosomal RNA; $V 4$ : hypervariable region 4.

\section{Acknowledgements}

We would like to thank Leslie Reed (DVM) from the Wildlife Rehabilitation Center of Minnesota, Gina Taylor (RVT) and Ali Crumpacker (MA) from the Fund for Animals Wildlife Center in California, Sara Childs-Sanford (DVM, MS) from the Janet L. Swanson Wildlife Health Center at Cornell University in New York, Heather Freeman (Wildlife Rehabilitator) from the Woodlands Wildlife Refuge in New Jersey, Emily Meredith (Wildlife Rehabilitation Manager) from PAWS Wildlife Center in Washington and Florina Tseng (DVM) from the Tufts Wildlife Clinic in Massachusetts for collecting samples. We would additionally like to thank Lindy McBride (PhD), Wei Wang (PhD), Jessica Wiggins (Lab Manager) and Edward Schrom (MA) from Princeton University for their support during library preparation, sequencing, data processing and analysis. 


\section{Authors' contributions}

ALD and BMvH designed the study. ALD and KNL conducted the laboratory work. ALD processed and analyzed the data. ALD prepared and revised the manuscript. All authors read and approved the final manuscript.

\section{Funding}

Funding for this study was provided by the American Society of Mammalogists Grants-in-Aid of Research program. This material is based upon work supported by the National Science Foundation Graduate Research Fellowship under Grant No. DGE1656466.

\section{Availability of data and materials}

All sequencing data analyzed in the present study is publically available through the NCBI Sequence Read Archive under BioProject PRJNA562927. Demultiplexed, paired-end fastq files are available for each sample (BioSamples SAMN12659808 to SAMN12659960) with SRA accession numbers: SRR10044131 to SRR10044283. Sample metadata is publically available through Additional file 1: Table S1.

\section{Ethics approval and consent to participate}

The Princeton University Institutional Animal Care and Use Committee reviewed and approved of all sample collection procedures (Princeton IACUC \#2084A-16).

\section{Consent for publication}

Not applicable.

\section{Competing interests}

The authors declare that they have no competing interests.

Received: 22 July 2019 Accepted: 16 September 2019

Published online: 16 October 2019

\section{References}

1. Hengge UR, Currie BJ, Jäger G, Lupi O, Schwartz RA. Scabies: a ubiquitous skin disease. Lancet Infect Dis. 2006;6:769-79.

2. Arlian LG, Morgan MS. A review of Sarcoptes scabiei: past, present and future. Parasites Vectors. 2017;10:297.

3. Bornstein S, Morner T, Samuel W. Sarcoptes scabiei and sarcoptic mange. In: Samuel WM, Pybus MJ, Kocan AA, editors. Parasitic diseases of wild mammals. Ames: lowa State University Press; 2001. p. 107-19.

4. Arlian LG. Biology, host relations, and epidemiology of Sarcoptes scabiei. Annu Rev Entomol. 1989;34:139-61.

5. Astorga F, Carver S, Almberg ES, Sousa GR, Wingfield K, Niedringhaus KD, et al. International meeting on sarcoptic mange in wildlife, June 2018, Blacksburg, Virginia, USA. Parasites Vectors. 2018:11:449.

6. Walton S, Holt D, Currie B, Kemp D. Scabies: new future for a neglected disease. Adv Parasitol. 2004;57:309-77.

7. Pence DB, Ueckermann E. Sarcoptic mange in wildlife. Rev Sci Tech. 2002;21:385-98.

8. Kołodziej-Sobocińska M, Zalewski A, Kowalczyk R. Sarcoptic mange vulnerability in carnivores of the Białowieża Primeval Forest, Poland: underlying determinant factors. Ecol Res. 2014;29:237-44.

9. Montecino-Latorre D, Cypher BL, Rudd JL, Clifford DL, Mazet JAK, Foley JE. Assessing the role of dens in the spread, establishment and persistence of sarcoptic mange in an endangered canid. Epidemics. 2019;27:28-40.

10. Nimmervoll H, Hoby S, Robert N, Lommano E, Welle M, Ryser-Degiorgis MP. Pathology of sarcoptic mange in red foxes (Vulpes vulpes): macroscopic and histologic characterization of three disease stages. J Wildl Dis. 2013;49:91-102.

11. Newman TJ, Baker PJ, Harris S. Nutritional condition and survival of red foxes with sarcoptic mange. Can J Zool. 2002;80:154-61.

12. Almberg ES, Cross PC, Dobson AP, Smith DW, Hudson PJ. Parasite invasion following host reintroduction: a case study of Yellowstone's wolves. Philos Trans R Soc B. 2012;367:2840-51.

13. Pence DB, Windberg LA. Impact of a sarcoptic mange epizootic on a coyote population. J Wild Manag. 1994;58:624-33.
14. Al-Sabi MNS, Halasa T, Kapel CMO. Infections with cardiopulmonary and intestinal helminths and sarcoptic mange in red foxes from two different localities in Denmark. Acta Parasitol. 2014;59:98-107.

15. Mörner T. Sarcoptic mange in Swedish wildlife. Rev Sci Tech. 1992;11:1115-21.

16. Gortázar C, Villafuerte R, Blanco J, Fernández-De-Luco D. Enzootic sarcoptic mange in red foxes in Spain. Z Jagdwiss. 1998;44:251-6.

17. Lindström ER, Andrén H, Angelstam P, Cederlund G, Hörnfeldt B, Jäderberg $L$, et al. Disease reveals the predator: sarcoptic mange, red fox predation, and prey populations. Ecology. 1994;75:1042-9.

18. Little SE, Davidson WR, Howerth EW, Rakich PM, Nettles VF. Diseases diagnosed in red foxes from the southeastern United States. J Wildl Dis. 1998:34:620-4.

19. Gosselink TE, van Deelen TR, Warner RE, Mankin PC. Survival and causespecific mortality of red foxes in agricultural and urban areas of Illinois. J Wildl Manag. 2007;71:1862-73.

20. Niedringhaus KD, Brown JD, Sweeley KM, Yabsley MJ. A review of sarcoptic mange in North American wildlife. Int J Parasitol Parasites Wildl. 2019;9:285-97.

21. Honda K, Littman DR. The microbiota in adaptive immune homeostasis and disease. Nature. 2016;535:75-84.

22. Buffie CG, Pamer EG. Microbiota-mediated colonization resistance against intestinal pathogens. Nat Rev Immunol. 2013;13:790-801.

23. Grice EA. The skin microbiome: potential for novel diagnostic and therapeutic approaches to cutaneous disease. Semin Cutan Med Surg. 2014;33:98-103.

24. Walton SF. The immunology of susceptibility and resistance to scabies. Parasite Immunol. 2010;32:532-40.

25. Shelley WB, Shelley ED, Burmeister V. Staphylococcus aureus colonization of burrows in erythroderma Norwegian scabies: a case study of iatrogenic contagion. J Am Acad Dermatol. 1988;19:673-8.

26. Swe PM, Fischer K. A scabies mite serpin interferes with complementmediated neutrophil functions and promotes staphylococcal growth. PLoS Negl Trop Dis. 2014;8:e2928.

27. Mika A, Reynolds SL, Pickering D, McMillan D, Sriprakash KS, Kemp DJ, et al. Complement inhibitors from scabies mites promote streptococcal growth-a novel mechanism in infected epidermis? PLoS Negl Trop Dis. 2012;6:e1563.

28. Swe PM, Zakrzewski M, Kelly A, Krause L, Fischer K. Scabies mites alter the skin microbiome and promote growth of opportunistic pathogens in a porcine model. PLoS Negl Trop Dis. 2014;8:e2897.

29. Fraser TA, Charleston M, Martin A, Polkinghorne A, Carver S. The emergence of sarcoptic mange in Australian wildlife: an unresolved debate. Parasites Vectors. 2016;9:316.

30. Rodrigues Hoffmann A, Patterson AP, Diesel A, Lawhon SD, Ly HJ, Elkins Stephenson C, et al. The skin microbiome in healthy and allergic dogs. PLOS ONE. 2014;9:e83197.

31. Bradley CW, Morris DO, Rankin SC, Cain CL, Misic AM, Houser T, et al. Longitudinal evaluation of the skin microbiome and association with microenvironment and treatment in canine atopic dermatitis. J Investig Dermatol. 2016;136:1182-90.

32. Kong HH, Oh J, Deming C, Conlan S, Grice EA, Beatson MA, et al. Temporal shifts in the skin microbiome associated with disease flares and treatment in children with atopic dermatitis. Genome Res. 2012;22:850-9.

33. Williams MR, Gallo RL. The role of the skin microbiome in atopic dermatitis. Curr Allergy Asthma Rep. 2015;15:65.

34. Wollina U. Microbiome in atopic dermatitis. Clin Cosmet Investig Dermatol. 2017:10:51-6.

35. Pence DB, Windberg LA, Sprowls R. The epizootiology and pathology of sarcoptic mange in coyotes, Canis latrans, from south Texas. J Parasitol. 1983;69:1100-15.

36. DeCandia AL, King JL, vonHoldt BM. Microbial dysbiosis and its implications for disease in a genetically depauperate species. bioRxiv. 2019. https ://doi.org/10.1101/653220.

37. Caporaso JG, Lauber CL, Walters WA, Berg-Lyons D, Lozupone CA, Turnbaugh PJ, et al. Global patterns of $16 \mathrm{~S}$ rRNA diversity at a depth of millions of sequences per sample. Proc Natl Acad Sci USA. 2011;108:4516-22.

38. Afgan E, Baker D, Batut B, Van Den Beek M, Bouvier D, Ech M, et al. The Galaxy platform for accessible, reproducible and collaborative biomedical analyses: 2018 update. Nucleic Acids Res. 2018;46:W537-44. 
39. Caporaso JG, Kuczynski J, Stombaugh J, Bittinger K, Bushman FD, Costello EK, et al. QIIME allows analysis of high-throughput community sequencing data. Nat Methods. 2010;7:335-6.

40. Bolyen E, Rideout JR, Dillon MR, Bokulich NA, Abnet CC, Al-Ghalith GA, et al. Reproducible, interactive, scalable and extensible microbiome data science using QIIME 2. Nat Biotechnol. 2019;37:852-7.

41. Callahan BJ, McMurdie PJ, Rosen MJ, Han AW, Johnson AJA, Holmes SP. DADA2: high-resolution sample inference from Illumina amplicon data. Nat Methods. 2016;13:581-3.

42. Rognes T, Flouri T, Nichols B, Quince C, Mahé F. VSEARCH: a versatile open source tool for metagenomics. PeerJ. 2016;4:e2584.

43. Vázquez-Baeza Y, Pirrung M, Gonzalez A, Knight R. EMPeror: a tool for visualizing high-throughput microbial community data. Gigascience. 2013;2:16.

44. Anderson MJ. A new method for non parametric multivariate analysis of variance. Austral Ecol. 2001;26:32-46.

45. DeSantis TZ, Hugenholtz P, Larsen N, Rojas M, Brodie EL, Keller K, et al. Greengenes, a chimera-checked 165 rRNA gene database and workbench compatible with ARB. Appl Environ Microbiol. 2006;72:5069-72.

46. Bokulich NA, Kaehler BD, Rideout JR, Dillon M, Bolyen E, Knight R, et al. Optimizing taxonomic classification of marker-gene amplicon sequences with QIIME 2's q2-feature-classifier plugin. Microbiome. 2018;6:90.

47. Mandal S, Van Treuren W, White RA, Eggesbø M, Knight R, Peddada SD. Analysis of composition of microbiomes: a novel method for studying microbial composition. Microb Ecol Health Dis. 2015;26:27663.

48. Morton JT, Sanders J, Quinn RA, Mcdonald D, Gonzalez A, Vázquez-baeza $Y$, et al. Balance trees reveal microbial niche differentiation. mSystems. 2017;2:e00162-16.

49. Altschul SF, Gish W, Miller W, Myers EW, Lipman DJ. Basic local alignment search tool. J Mol Biol. 1990;215:403-10.

50. Letunic I, Bork P. Interactive tree of life (iTOL) v3: an online tool for the display and annotation of phylogenetic and other trees. Nucleic Acids Res. 2016;44:W242-5.

51. DeCandia AL, Dobson AP, vonHoldt BM. Toward an integrative molecular approach to wildlife disease. Conserv Biol. 2018;32:798-807.

52. Hauffe $\mathrm{HC}$, Barelli $\mathrm{C}$. Conserve the germs: the gut microbiota and adaptive potential. Conserv Genet. 2019;20:19-27.

53. Trevelline BK, Fontaine SS, Hartup BK, Kohl KD. Conservation biology needs a microbial renaissance: a call for the consideration of hostassociated microbiota in wildlife management practices. Proc R Soc B. 2019;286:20182448.

54. Bonder MJ, Kurilshikov A, Tigchelaar EF, Mujagic Z, Imhann F, Vila AV, et al. The effect of host genetics on the gut microbiome. Nat Genet. 2016:48:1407-12.

55. Rothschild D, Weissbrod O, Barkan E, Kurilshikov A, Korem T, Zeevi D, et al. Environment dominates over host genetics in shaping human gut microbiota. Nature. 2018;555:210-5.

56. Brucker RM, Bordenstein SR. Speciation by symbiosis. Trends Ecol Evol. 2012;27:443-51.

57. Brooks AW, Kohl KD, Brucker RM, van Opstal EJ, Bordenstein SR. Phylosymbiosis: relationships and functional effects of microbial communities across host evolutionary history. PLoS Biol. 2017;14:e2000225.

58. Knowles SCL, Eccles RM, Baltrūnaitè L. Species identity dominates over environment in shaping the microbiota of small mammals. Ecol Lett. 2019;22:826-37.

59. Nishida AH, Ochman H. Rates of gut microbiome divergence in mammals. Mol Ecol. 2018;27:1884-97.

60. Whitehall J, Kuzulugil D, Sheldrick K, Wood A. Burden of paediatric pyoderma and scabies in North West Queensland. J Paediatr Child Health. 2013:49:141-3.

61. McCarthy JS, Kemp DJ, Walton SF, Currie BJ. Scabies: more than just an irritation. Postgrad Med J. 2004;80:382-7.
62. Bergström FC, Reynolds S, Johnstone M, Pike RN, Buckle AM, Kemp DJ, et al. Scabies mite inactivated serine protease paralogs inhibit the human complement system. J Immunol. 2009;182:7809-17.

63. Mika A, Reynolds SL, Mohlin FC, Willis C, Swe PM, Pickering DA, et al. Novel scabies mite serpins inhibit the three pathways of the human complement system. PLoS ONE. 2012;7:e40489.

64. Bannoehr J, Franco A, lurescia M, Battisti A, Fitzgerald JR. Molecular diagnostic identification of Staphylococcus pseudintermedius. J Clin Microbiol. 2009:47:469-71.

65. Fazakerley J, Nuttall T, Sales D, Schmidt V, Carter SD, Hart CA, et al. Staphylococcal colonization of mucosal and lesional skin sites in atopic and healthy dogs. Vet Dermatol. 2009;20:179-84.

66. Bannoehr J, Guardabassi L. Staphylococcus pseudintermedius in the dog: taxonomy, diagnostics, ecology, epidemiology and pathogenicity. Vet Dermatol. 2012;23:253-e52.

67. Ngo J, Taminiau B, Fall PA, Daube G, Fontaine J. Ear canal microbiota-a comparison between healthy dogs and atopic dogs without clinical signs of otitis externa. Vet Dermatol. 2018;29:425-e140.

68. Pompilio A, De Nicola S, Crocetta V, Guarnieri S, Savini V, Carretto E, et al. New insights in Staphylococcus pseudintermedius pathogenicity: antibiotic-resistant biofilm formation by a human wound-associated strain. BMC Microbiol. 2015;15:109.

69. Sasaki T, Kikuchi K, Tanaka Y, Takahashi N, Kamata S, Hiramatsu K. Methicillin-resistant Staphylococcus pseudintermedius in a veterinary teaching hospital. J Clin Microbiol. 2007:45:1118-25.

70. Weese JS, van Duijkeren E. Methicillin-resistant Staphylococcus aureus and Staphylococcus pseudintermedius in veterinary medicine. Vet Microbiol. 2010;140:418-29.

71. Durvasula RV, Sundaram RK, Kirsch P, Hurwitz I, Crawford CV, Dotson E, et al. Genetic transformation of a Corynebacterial symbiont from the Chagas disease vector Triatoma infestans. Exp Parasitol. 2008;1 19:94-8.

72. Rudolf I, Mendel J, Šikutová S, Švec P, Masaříková J, Nováková D, et al. 165 rRNA gene-based identification of cultured bacterial flora from host-seeking Ixodes ricinus, Dermacentor reticulatus and Haemaphysalis concinna ticks, vectors of vertebrate pathogens. Folia Microbiol. 2009;54:419-28.

73. Rowe ML, Whiteley PL, Carver S. The treatment of sarcoptic mange in wildlife: a systematic review. Parasites Vectors. 2019;12:99.

74. Thomas J, Peterson GM, Walton SF, Carson CF, Naunton M, Baby KE. Scabies: an ancient global disease with a need for new therapies. BMC Infect Dis. 2015;15:250.

75. Murray M, Edwards MA, Abercrombie B, Cassady C, Clair S. Poor health is associated with use of anthropogenic resources in an urban carnivore. Proc R Soc B. 2015;282:20150009.

76. Murray MH, St. Clair CC. Predictable features attract urban coyotes to residential yards. J Wildl Manag. 2017;81:593-600.

77. Carricondo-Sanchez D, Odden M, Linnell JDC, Odden J. The range of the mange: spatiotemporal patterns of sarcoptic mange in red foxes (Vulpes vulpes) as revealed by camera trapping. PLoS ONE. 2017;12:e0176200.

\section{Publisher's Note}

Springer Nature remains neutral with regard to jurisdictional claims in published maps and institutional affiliations.

Ready to submit your research? Choose BMC and benefit from:

- fast, convenient online submission

- thorough peer review by experienced researchers in your field

- rapid publication on acceptance

- support for research data, including large and complex data types

- gold Open Access which fosters wider collaboration and increased citations

- maximum visibility for your research: over $100 \mathrm{M}$ website views per year

At BMC, research is always in progress.

Learn more biomedcentral.com/submissions 